\title{
INHALTSVERZEICHNIS
}

Vorbemerkungen $\ldots \ldots \ldots \ldots \ldots \ldots \ldots \ldots \ldots \ldots$

TEIL I

\section{Le siècle de Louis XIV}

Kapitel I Horizonte $\ldots \ldots \ldots \ldots \ldots \ldots \ldots \ldots \ldots . \ldots . \ldots . \ldots$

Die Veränderung der Weltkarte. Durchbruch der Staatsraison. Das Jahrhundert der religiösen Bewegungen: Port Royal, Gallikanismus, quietistische Mystik, die Jesuiten. Der Einbruch naturwissenschaftlichen Denkens und Forschens. Das Jahrhundert Descartes' und Fermats, Galileis und Pascals. Die Literatur: eine Renaissance der Antike. Die Krise des europäischen Bewußtseins. Die Klassik: Verehrung der Antike; Glaube an die Vernunft: Nachahmung der Natur; der Zug zum Grandiosen und Majestätischen: Versailles. - Die Kehrseite der Medaille: Korruption der höfischen Welt und Armut des Volkes. - Das Wirtschaftsleben im Jahrhundert Colberts. Das demographische Bild. Die Widersprüche des Jahrhunderts. Im Zwielicht zweier Epochen.

Kapitel II Staat und Gesellschaft im Frankreich des XVII. Jahrhunderts. Von Richelieu zu Ludwig XIV. ..

Richelieu. Frankreich auf dem Wege zur militärischen, politischen und kulturellen Hegemonie über Europa. Richelieus „Politisches Testament“. Grundsätze der Staatsraison. Die Gründung der „Académie Française“.

Mazarin. Die Ereignisse der Fronde.

Ludwig XIV. Das Schlüsselwort seiner Staatsführung: Règlement. Reglementierung des Lebens, des Staates, der Verwaltung, der Wirtschaft, des Heerwesens, der Kirche, der Kultur. Abstieg des Adels und Aufstieg des Bürgertums. Colbert und der Merkantilismus. Gelenkte Wirtschaft. Die Kirchenpolitik. - Das vielschichtige Phänomen der Preziosität. Die Salons, ihre Intérieurs, die kulturelle Rolle der Preziösen.

Kapitel III Die Welt der Mathematiker Philosophen und Kirchenfürsten .................... 55

Descartes. Ein Weltmann zieht die Bilanz der Wissenschaften. Die 4 provisorischen Verhaltensweisen des Menschen: eine weltmännische Empfehlung und Philosophie. Das cogito ergo sum. Die zwei Lager seiner Gegner. 
Pascal. Ein mathematisches und religiöses Genie. Etappen seines Lebenswegs: vom mathematischen Wunderkind über ein mondänes Leben zur Nachfolge Christi. - Die Konstruktion der Rechenmaschine. - Die Krise und die Nacht der Offenbarung. - Von der Lehre der Herren von Port Royal. Die Verteidigung der Jansenisten. "Lettres provinciales". Die "Pensées“: Apologie des Christentums. Pascal bekämpft Descartes. Von dem politischen Problem Christ und Welt.

Bossuet. Der gallikanische Kirchenfürst und Kanzelredner. Der Dreiklang seiner Thematik. Ein pädagogisches Geschichtswerk ad usum Delphini: der

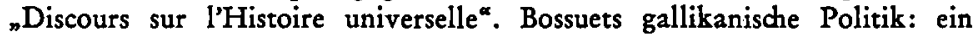
Schaukelspiel zwischen Papst und König. Bossuet: "C. M. P.“ - Das Scheitern der Reunionsverhandlungen zwischen Katholiken und Protestanten. Bossuets Kanzelberedsamkeit. Die Begängnisse der Großen des Königreichs als heilige Parade.

Fénelon. Ein mystisches Christentum. "Doktor, Bischof und Grandseigneur". Der "Télémaque", ein Bildungsroman mit politischen und sozialkritischen Tendenzen ad usum Delphini - des „petit dauphin“. - Die Kühnheit des „Briefes an Ludwig XIV.“. - Die Affäre des Quietismus. - Fénelons Stellung im „Streit der Alten und Modernen ${ }^{\alpha}$. Die Krise der französischen Kultur am Ausgang des Jahrhunderts: ein dramatischer Vorgang in 2 Akten.

Kapitel IV Die Welt des Theaters ............. 108

1. Die französische Theaterkultur: ein künstlerisches und soziales Ereignis. Spielhäuser, Spielsäle, Spielzeiten und Inszenierungspraxis. Der Einfluß Vitruvs. Der Bereich der Oper. Die Rolle Mazarins. Vom Wesen der "représentation en musique ${ }^{\alpha}$ und die geschichtliche Perspektive der Oper. Camberts Memorandum für den König und Perrins Ideen für ein Musikschauspiel. Die Gründung der "Académie d'Opéra". Der Aufstieg Lullis am Hofe Ludwigs XIV. Ein Urteil Goethes über die Bedeutung Lullis. - Das Ballett. Gründung der "Académie de Danse ${ }^{\alpha}$ 1661. Der Sonnenkönig tanzt den Jupiter. Bürgerliche Berufstänzer und adlige Amateurtänzer. Der Luxus der Kostüme. Von den Bühnenbildern: eine Wunderwelt der Kulissen.

2. Die andere Hemisphäre der Theaterwelt: die Klassiker des Worttheaters: Corneille, Wegbereiter des klassischen Theaters in Frankreich. Grundfragen der Dramaturgie. - Vom ${ } \mathrm{Cid}^{\alpha}$ und anderen Meisterdramen. Die "theoretische Salbaderei“. Corneilles Vorbehalte gegenüber den Alten. Ein Blick auf Aristoteles. Kartesianismus - Stoizismus - christliche Moral. - Racine, Höhepunkt des klassischen Theaters. Die Berührung mit der griechischen Kultur. Die Bildung seines literarischen Geschmacks und seiner poetischen Sensibilität. Zwielichtigkeit seines Charakters. Frauen um Racine. Der Aufstieg zum Historiographen des Königs. - Versöhnung mit Port Royal. - Racines Bühnenwerk auf den Fundamenten der Corneilleschen Dramaturgie. Ein Urteil Giraudoux'. - Racines Themen. Die Transkrip- 
tion der Vorlagen in die zeitgebundene Empfindsamkeit. Vom Aufbau seiner Tragödien und dem Fundamentalsatz seiner Asthetik. Die philosophisch-religiöse Schicht seiner Tragödienwelt. Das Wunderwerk der „Phèdre ${ }^{\alpha}$. Die drei Antlitze des Racineschen Fatums. Griechische Schicksalsidee und jansenistische Gnadenwahl. - Molière, „premier farceur de

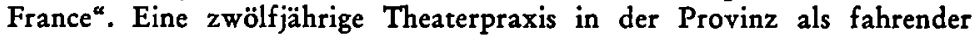
Schauspieler, Autor und Schauspieldirektor. Einzug in Paris. Aufstieg am Hofe. - Ein Mime italienischen Stils. Auf dem Grenzpfad zwischen Komödie und Tragödie. Das Ende einer großen Schauspielerkarriere. „Le dieu des ris" und seine "grande règle de toutes les règles“. Ein Bekenntnis Louis Jouvets zu Molière. Die Bedeutung der Farce und die sprachlichen, psychologischen und mimischen Elemente der vis comica. Ist es schwerer, Komödien oder Tragödien zu schreiben? - Das Bild der Zeit und Gesellschaft im Spiegel des Molièreschen Oeuvre. Molières Kampf um die Befreiung des Menschen aus sozialen, religiösen und moralischen $Z$ wängen. Der Einspruch der Kirche gegen den Komödianten und sein Werk.

3. Lafontaine: ein Welttheater in Miniaturszenen. Der Fabeldichter. Was ist eine Fabel? Lafontaines Quellen und die Originalität seiner Fabelwelt. Ein „hundertaktiges Drama“ von Göttern, Menschen, Tieren. - Der Antikartesianismus: ein Aspekt der libertinistischen Bewegung um Gassendi. Lafontaines Sympathien für Epikur, Lukrez, Gassendi. Die Fabeln als szenische Gudkkastenbildchen der sozialen Hierarchie: König - Geistlichkeit - Adel - Bürgertum - Volk.

Kapitel V Das Epiphaniasfest der Musen ......... 170 Nicolaus Boileau: Gesetzgeber der Klassik post festum. Sein ${ }_{n}$ Art poétique“ als corpus doctrinale des hohen Kunstgeschmacks. Das Studium der antiken Theoretiker von Aristoteles bis Longinos, und der Umgang mit den Zeitgenossen Racine, Molière, Lafontaine. - Die antihistorische Perspektive. Boileaus Organ für griechischen Kunstverstand. Das "Sublime“ als Begnadung des Dichters. Zwei Beispiele. Der entscheidende Einfluß von Horaz. Ein Dialog zweier kongenialer Geister. Simile simili cognoscitur. „Der wahre Inhalt ist die Form ${ }^{\text {“ }}$. Berechnung der Werkstrukturen. Gleidhgewicht und Ebenmaß. Boileaus Kritik im kritischen Spiegel der heutigen Zeit. Der „homme du juste milieu“. Eine neue Zeit bricht an.

\section{TEIL II}

\section{Im Lichte der Aufklärung}

\section{Kapitel I Geist, Kunst, Gesellschaft im Frankreich des} XVIII. Jahrhunderts .................. 185

"Was ist Aufklärung? “ Das Ferment der Naturwissenschaften in der Epoche der mathesis universalis. - Das Bild der Gesellschaft im Wandel der 
Schönen Künste. Vom Barock zum Rokoko. Vom Geist der französischen Musik in der Gesellschaft des 18. Jahrhunderts. Die Opernkultur im Wandel der Zeit. Von der Régence zur Revolution.

\section{Kapitel II Die vier Wegbereiter der modernen Zeit ..... 230}

Montesquieu: ein humanistischer Weltbürger und die Weisheit politischen Denkens. Die 3 Etappen seines Weges: die "Lettres persanes" "chers Romains" - sein "De l'Esprit des Lois“. Fragen der Regierungsformen; Teilung der Gewalten; von der Wirkung des Buches.

Voltaire: die Schlagkraft des befreiten Geistes. Ein Leben des Wortes und der Tat. Der Historiker: Geschichte im Spiegel der Aufklärungsphilosophie. - Der Pbilosoph als Kritiker von Pascal, Leibniz, Rousseau. Der Theatromane: Theater als Stätte der Erziehung, als soziale Institution und als Kunstgattung. Voltaire und Shakespeare. Ein rüdkwärtsgewandter moderner Geist. Nietzsche über Voltaire.

Rousseau: Bewußtsein und Gewissen einer zukünftigen Zeit und Gesellschaft. Die Universalität eines Originalgenies: Pädagoge, Soziologe, Philosoph, Psychologe, Botaniker, Musiker, Romancier. - Aus seinem Leben. Die beiden "Discours": Ouvertïre eines Lebenswerks. Der "Contract social“" die theoretische Ausgleichsrechnung des Gesellschaftsvertrags und die Begründung einer totalen Demokratie. Der "Emile“: Probleme einer modernen Erziehung und deren Methoden. Das zentrale Kapitel des religiösen Glaubensbekenntnisses. - "Julie ou la Nouvelle Héloise“. Ein Liebesroman der Weltliteratur. Biographische Hintergründe. Eine literaturgeschichtliche Perspektive: Bindeglied $z$ wischen dem alten englischen Romancier Richardson und dem jungen deutschen Verfasser des "Werther ${ }^{\alpha}$.

Diderot: tot artes et tantae scientiae: der Enzyklopädist und die Fülle der Welterfahrung. - Vom Wandel seines Weltbildes. Uber die Künste. Goethe im Gespräch mit Diderot. Die "Salons“ und "Rameaus Neffe". Der bürgerliche Dramaturg: zwischen Tragödie und Komödie. Verbindung mit Lessing. - Die Encyclopédie. Am Vorabend der „ungeheuren Weltveränderungen".

Kapitel III Das Ende des Ancien Régime .......... 342

Auf dem Weg zur Revolution. Vorspiel auf dem Theater. Mercier und Beaumarchais. - "Qu'est-ce que le tiers état?" Von Sieyès, seiner Persönlichkeit, seinen Schriften, seiner Wirkung. Die "Eloquence révolutionnaire". Die Etappen der Revolution auf einer Parabel. - Von Sieyès und Mirabeau über Barnave und Condorcet zu Danton, Robespierre und SaintJust. Nachspiel und Auftakt zu der Tragödie Zweitem Teil: Napoléon. 
TEIL III

\section{Der Torso des XIX. Jahrhunderts}

Kapitel I Auftakt zum neuen Jahrhundert .......... 379

Drei Schlüsselfiguren im dramatischen Spannungsfeld zwischen 1789 und 1830: Chateaubriand - Germaine de Staël - Napoléon.

Kapitel II Was heißt Romantik, und was ist ein Romantiker? 396 Von einigen Aspekten der romantischen Bewegung in Frankreich. Fünf Kardinalfragen: 1. Die Wege der Romantiker zu Gott. - 2. Die Romantiker vor und in der Gesellschaft und Geschichte. Saint-Simon, Enfantin, Fourier. - 3. Romantische Aspekte der "Natur“. - 4. Elemente romantischer Weltbilder: Neuplatonismus, Mystik, Magie. Im Bann des Märchens. - 5. Die Kunst: romantische Aktivität par excellence. - Die Ironie und der Weg zum Surrealismus. - „Was für eine neuartige Wissenschaft!“

Kapitel III Die Künste im Zeitalter der Romantik ...... 411 Eigenständigkeit und Symbiose. Die Architektur: Der Kirchentempel der Madeleine; Warenhäuser von Paris; die Halle au blé. Romantische Baugesinnung. Exotismen. Architektonische Zukunftsphantasien dreier Originale. - Die Bildbauerkunst David d'Angers: "tous les nobles aspects de la figure humaine“. - Die Malerei. Vom Klassizismus zur Romantik. Louis David: das politische Engagement eines großen Malers. Ingres: die Zeichnung als "Redlichkeit der Kunst". Drei Grundsätze seiner Ästhetik. Delacroix: „die schönste Palette Frankreichs". Der Maler in Baudelaires Urteil: „passionnément amoureux de la passion“. Die Sprache der Modernität. - Die Musik. Drei musikalische Ereignisse im Frankreich der Restauration. 1. Beethoven und Weber im Pariser Musikleben. - 2. Die "Große Oper“. Musik, Gesellschaft und Politik. Aus Wagners „Erinnerungen an Auber". Rossini, Meyerbeer. "Die Religion Mozarts, Gludks, Beethovens." - 3. Das Phänomen Berlioz oder der Einbruch der europäischen Dichtung in die Thematik der symphonischen Musik. Die "Symphonie fantastique und Schumanns Analyse der Tondichtung. Berlioz-Liszt-Wagner: „Wir drei Kerle. ${ }^{\alpha}$

Kapitel IV Die Weltgültigkeit der französischen Lyrik des XIX. Jahrhunderts. Von der Romantik zum Symbolismus .....................440

1. Der Parnaß zwischen Romantik und Symbolismus

Die soziale Misere der Dichter und Künstler. Théodore de Banville, ein Lehrmeister der Kompositionstechnik. Was ist Poesie? Geist und Form. Realismus und Supranaturalismus. Der Zirkusclown vor metaphysischem Hintergrund. Auf dem Wege zum Expressionismus: „Der Maler muß nicht den Baum darstellen, sondern ihn sichtbar machen." - Ein Wort über Leconte de Lisle. 
2. Die großen Vier der zweiten Jahrhunderthälfte

Baudelaire. Eine poetische Mission. Die Metaphysik des Dandytums. „Le dernier éclat d'héroisme dans les décadences." Das Lob der Schminke. Die Baudelairesche Spiritualität. Zwischen Philosophie und der Dichtung des Bizarren und Insoliten. Der „Rêve Parisien“. Romantik und Surrealismus. Mallarmé. Poesie in der Höhenluft einer entdinglichten Welt. Die Metaphysik des Sagens und Schweigens. Dunkelheit. Der esoterische Charakter des Dichtens. Das ideelle Nichts. Sprachliche Askese als Geheimnis weltweiter Wirkung.

Verlaine. Der „Saturn-Mensch“. Größe und Elend eines Dichterlebens. Faszinosum der Musik. Von der Technik der Verskunst. Poète maudit oder der hl. Franziskus?

Rimbaud. „Mehr als eine literarische Angelegenheit." Das trunkene Schiff: Vorentwurf eines Lebens. Von der Phantasie der Rimbaud-Deuter. Entfesselung der Sinne - Revolte gegen die Tradition - der Argonautenzug zum Goldenen Vlies der Sprache. "Ich erfand die Farbe der Vokale ${ }^{\text {" }}$ "Magische Sophismen" - "Halluzination der Worte" . - Die törichte Jungfrau und ihr höllischer Gemahl. Eingeblendet: Le Comte de Lautréamont. Proleptischer Surrealismus: „Die Begegnung einer Nähmaschine und eines Regenschirms auf einem Seziertisch. “ Aufruf zur Revolte. - Rimbaud: Heide oder Christ? Der Schatten Nietzsches. - Eine neue sprachliche Architektur ohne Gravitationszentrum.

3. Vom Symbolismus

Die Generation der „Décadents“. Der „Idéoréalisme : ein symbolistisches Phänomen des poetischen Platonismus. Jean Moréas. Das Manifest des Symbolismus. Aus dem "Ermitage“ von Mazel. - Die „Ecole romane ${ }^{\alpha}$. Das geistig-soziale Klima der 90 er Jahre. - E. A. Poe und Richard Wagner im Kunst- und Geistesleben der Symbolistengeneration. Poe-BaudelaireMaeterlindk. Grand-Guignol. Von der Schauernovelle zum Schauertheater. - Poe-Debussy. Eine Transfiguration dichterischer Erlebnisse in eine neue Syntax der Musik. Die Poe-Maeterlindk-Munch-Atmosphäre der französischen Dichtung, Malerei und Musik um die Jahrhundertwende. - Drei Wege zu einer Analyse Debussys.

Kapitel V Die vier großen des französischen Romans. Ein Gesellschaftsbild des XIX. Jahrhunderts ...... 490

1. Stendhal. Zwischen Vergangenheit und Zukunft. Das Jugenderlebnis Napoleon. Aristokrat und Plebejer. Der Leser Napoleons und Fouriers. ${ }_{n}$ Rot und Schwarz ${ }^{\alpha}$ - symbolische Farben auf dem gesellschaftlichen Hintergrund von 1830. Julien Sorel: die Fatalität der condition humaine.

2. Balzac. Seine Statue. Ein Koloß unter den Epikern. Potenzierte Willensenergie. Der phänomenale Gedächtnisspeicher. Die visionäre Phantasie. Divina Commedia und Comédie humaine. Von der Uberwelt Swedenborgs zur Unterwelt von Paris. Der Buffon der französischen Gesellschaft. Beobachtung und Analyse. Der Tanz ums goldene Kalb. Die kapitalistische 
Tragikomödie: der alte Grandet. Balzac: Historiker und Sekretär der französischen Gesellschaft. Die Geistesfamilie Balzacs im 19. Jahrhundert.

3. Flaubert. Der normannische Recke im härenen Gewand. Nietzsche durchleuchtet Flaubert. Der Wille zur Selbstüberwindung. Sein Künstlerengagement. Zu Flauberts Stil. Die 4 großen Werke: Madame Bovary: Fatalität und Tragik im Schatten Schopenhauers. Salammbô: Problem eines historischen Romans. Zwischen Chateaubriand und Malraux. L'Education sentimentale: Die Illusion einer Generation oder wie man das Leben verfehlt. Roman ohne Helden. La Tentation de Saint-Antoine: Ein metaphysisches Drama zwischen Breughel und Dali. Vom "Wilhelm Meister" zum "Faust". Spuk, Magie, Dämonenwelt und Menschenschicksal. Götterdämmerung und Wissenschaftsgläubigkeit. Christus in der Sonnenscheibe.

4. Zola. Eine neue Generation von Shriftstellern, Malern, Musikern. Das Unternehmen der Rougon-Macquart: eine naturalistische Sozialgeschichte Frankreichs im Zweiten Kaiserreich. Wissenschaft und Literatur. Von der experimentellen Methode. Die Steigerung des naturalistischen Romans ins Mystische. Drei Beispiele: Nana, Au Bonbeur des Dames, Germinal, oder: die babylonische Ischtar, Magie des Warenhauses, Zukunftsschau der "roten Revolution “. Von der literarischen Technik des Leitmotivs. Zola und Wagner. - Der Utopist der Vier Evangelien. Die Tetralogie und der Hintergrund der Dreyfusaffaire. Größe und Grenzen seines Werkes. Vom Geist zur Tat.

\section{Kapitel VI Positivistische Philosophie, Experimentalwissen- schaft, deterministisches Weltbild. Von Auguste} Comte über Claude Bernard zu Hippolyte Taine 537

1. Auguste Comte. Ursprung der Comteschen Philosophie: "Diese Klasse arbeitender Menschen...* - Die 4 bestimmenden Mächte: der Katholizismus des Kleinbürgertums; die Lektüre Condorcets; als Sekretär von SaintSimon; die Ecole polytechnique. - Die Stellung der Mathematik. „Soziale Physik *. Antikommunismus. - Die Lehre von den 3 Entwidklungsstadien der Menschheit. Der Sprung in die Transzendenz. Der Soziologe als Pontifex maximus einer neuen Menschheitsreligion. - Die Ambiguität der Comteschen Persönlichkeit und ihres Werkes.

2. Claude Bernard. Theoretiker der Experimentalwissenschaft. Von Bernard zu Plandk und Heisenberg. - Auf dem Wege zur wissenschaftlichen Medizin. Die Experimentalmethode. Der dreifache Weg: Die Macht der Phantasie; Experimentator gegen Scholastiker; das Credo des Determinismus. - Die Ablösung der persönlichen Autorität eines Forschers durch die "unpersönliche Form ${ }^{\alpha}$ einer Gemeinschaftsarbeit. Hugo: „Die Kunst ist das Ich, die Wissenschaft das Wir." Der Weg zur Allmacht des Menschen. Erwartung und Grenzen.

3. Hippolyte Taine. Der Klassiker des Determinismus. In der Nachfolge Claude Bernards. Die „einfache Formel “ seiner Methode. Ein Brückenschlag zu Robert Mayer: "causa aequat effectum. " Taines "Chemie" als 
psychologische und soziologische Analyse. Die Entkräftung theologischer

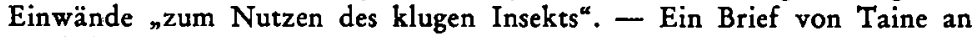
Berthelot. Naturwissenschaft als neue Religion. - Taines „Philosophie de l'Art". Der positivistische Kulturphilosoph vor dem Phänomen der Kunst. Die methodische Erhellung eines Kunstwerks. Von der Kunst der Imitation zur Kunst der Expression. Die Wachstumsbedingungen aller Kunst: Klima, Temperatur, öffentliche Meinung. - Die großen Epochen abendländischer Zivilisation und ihre Kunsterscheinungen. Von der Antike zum Bild des "modernen" Menschen. Umschau und Vorschau.

\section{TEIL IV}

\section{$\mathrm{Zu}$ neuen Ufern}

Kapitel I Die französische Gesellschaft im XX. Jahrhundert Daten - Ereignisse - Tendenzen ......... 569

1. Von der Müdigkeit des Fin de siècle zur Euphorie der Jahrhundertwende. Die „belle époque“. Ein verblühender Traum. - Die Risse im Gefüge des Staatsbaus. Drei Charakterzüge der "Modernität“: „Die Anatomie des Seelenlebens ${ }^{\alpha}$ — "das Ballspiel mit dem All“ — „die funkelnde Metapher".

2. Das Erwachen. Der Erste Weltkrieg und seine Folgen. Arbeitgeber und Arbeitnehmer. Wandel des Wirtschaftslebens und des politischen Klimas. Neue Hoffnungen: der Völkerbund. "Le rapprochement franco-allemand ${ }^{*}$. 3. Der Wandel um 1930. Politische Vorspiele im Schatten der Zweiten Katastrophe des "drôle de guerre“. Die Spaltung der Geister.

4. Nach dem Zweiten Weltkrieg. Die "Anti"-Tendenzen. Das alpha privativum der "Aliteratur" und die Literatur des Existenzialismus, der Absurdität und des politischen und philosophischen Ernstes.

5. Die neue französische Gesellschaft. Die Bauernschaft. Die ,révolution silencieuse ${ }^{\alpha,}$ die "journée des barricades" und die "révolution des tracteurs". Aktion und Reaktion im landwirtschaftlichen Sektor. Austritt aus der "splendid isolation". - Die Arbeiterklasse. Eine "nouvelle classe ouvrière". Vom Industriemuseum zur Automation. Unternehmertum und Arbeiterschaft. Entromantisierung des Streiks. Neue Gegensätze. Reformen oder Revolution? Von der Mentalität des französischen Arbeiters. - Der Mittelstand. Seine Vielschichtigkeit. Die "cadres supérieurs" und die "cadres moyens“. Ein neuer Angestelltentyp. Der Manager als Auftragsunternehmer.

Kapitel II Vier klassische Profile am Eingang zum Jahrhundert .......................... 591

1. André Gide. Ein "kontradiktatorisches Produkt". Seine Welten. Von der Klassik und Romantik. Der J. S. Bach- und Chopinspieler. Das 
Diptychon des "Immoraliste" und der "Porte étroite“. Der Jux von den "Verliesen des Vatikans". Auf dem Weg zu einer neuen Romankonzeption: "Die Falschmünzer." - Nach Moskau. "Ein Ritter ohne Furcht und Tadel." Die Enttäuschung. Der chinesische Weise.

2. Paul Valéry. "Lascia la poesia e studia la matematica." Seine 3 Masken: Narkissos-Leonardo-M. Teste. - Die folgenreichste Geistestat in der Geschichte der Menschheit: die griechische Mathematik. - „Alle großen Dichter werden am Ende Kritiker." - Der Blitz in seinem Leben: Mallarmé. Die Konzeption der Valéryschen Dichtung. Am Kreuzungspunkt von Dichtung und Musik. Der emotionale und intellektuelle Weg zur Musik. Der „Biß des Neides“. "Amphion": Vom Bau des Tempels oder das Mysterium der Musik. Für oder gegen Dionysos? Nachklänge Nietzsches. Valéry und die Malerei. Vom "epischen Zeitalter der Malerei“ und dem Verhältnis des Dichters zur zeitgenössischen Kunst. Eine Parallele: Thomas Mann und Paul Valéry.

3. Marcel Proust. Die soziale Welt Marcel Prousts. Ihre Grenzen. Erinnertes und erinnerndes Ich. Die zerstörende Zeit und die bewahrende Erinnerung. Ein Geheimnis des keltischen Aberglaubens. - Strukturelemente seiner "Ring “-Komposition. Bekenntnis zu John Ruskin und den Leiden eines Künstlerdaseins: „cette famille magnifique et lamentable qui est le sel de la terre“. - Die Schöpfung der Welt aus einer Tasse Tee. - Die Thematik. Seine Romankunst als impressionistische Prozedur des Zerlegens und Zusammensetzens. Die platonische Erfahrung der intelligiblen Welt durch die Musik: „la petite phrase de Vinteuil“. Die Doppelerfahrung: Desillusion und Weltentfremdung - Glüdkseligkeit einer mystischen Gewißheit in der wiedergewonnenen Zeit. - "Die wahre Kunst hat nichts mit Proklamationen zu tun; sie erfüllt sich in der Stille."

4. Paul Claudel. Ein poeta sui generis. Im Würgegriff der Zeit. Rebellion und Bekehrung. Eine Konfrontation Gide - Claudel. - Das Argernis. Der Doppelsinn des „Katholischen“. Claudels Meister. Erlösungsmythen als Themen seiner Dramatik: Verwandtschaft mit Wagners Thematik. - Die Erfüllung Schlegelscher Theorien in Claudels Dramaturgie. - Sein theozentrisches Weltbild. Die dreifache Dimension seiner Dichtung. Sublimes Geschwätz oder tiefsinnige Symbolik? Der interpres deorum. Der "Soulier de Satin ${ }^{\alpha}$ aus dem Geist der spanischen Dramaturgie der Habsburg-Ära. Der katholische Zuschnitt eines faustischen Weltendramas. - Rückblidk auf die vier Klassiker des Jahrhunderts: Literatur und Dichtung im Bann der Künste und der Musik.

\section{Kapitel III Aspekte der französischen Malerei. Vom Realis-} mus zum Surrealismus ...............6 654

1. Zwischen Romantik und Impressionismus. Der soziale und politische Aspekt der Malerei um 1850. Daumier oder die comédie humaine eines Karikaturisten. - Millet: ein bäuerlicher Realismus und romantischer Sentimentalismus. - Courbet: ein sozialistischer Realist. - Corot: ein roman- 
tischer Maler unter den Wegbereitern des Impressionismus. Valéry und Corot: Musik der Corotschen Malerei.

2. Vom Impressionismus. Der Ärger mit der Ecole des Beaux-Arts. Vorläufer der Impressionisten. Von der Höhlenmalerei zur englischen Romantik. - Vom Wesen des Impressionismus. Maler über ihre Kunst. Sisley:

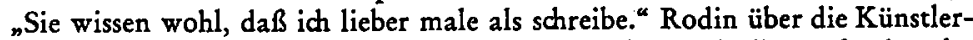
psyche. Kunstkritiker vom Fach über die Maler und die Malweise des Pleinairismus.

3. Vom Fauvismus zum Kubismus. Der "fauvisme" in der Nachbarschaft des deutschen Expressionismus. Nabi-Gruppe und Jugendstil. Die Geburt einer neuen Malerei um 1900. Drei Aussagen von Kirchner, Matisse, Klee. - Drei Daten im ersten Dezennium des neuen Jahrhunderts: 1. Datum: Hugo von Hofmannsthals Erlebnis der Retrospektive von 1901. Vincent van Gogh; 2. Datum: das annus mirabilis 1905. Maurice Denis in der "Ermitage" und Matisse: "Wir sind nicht Herr über unser Schaffen. Es ist uns auferlegt." 3. Datum: 1907: Picassos „Demoiselles d'Avignon“. Der Kubismus als Anti-Impressionismus. Seine wesentlichen Phasen. Apollinaire: Verkünder der neuen Bewegung. - Ein Blick auf die Ahnenreihe der Kubisten. "Wer Cézanne versteht, fühlt den Kubismus vor." - Vom Wesen kubistischer Malerei und von einigen Theorien. Eine konzeptionelle, intellektuelle, geistbeladene Kunst. "Cubisme analytique und "cubisme synthétique". Bezüge zur Musik und Mathematik. Braque: "Die Sinne entformen, der Geist formt." Apollinaire: „So gehen wir einer völlig neuen Malerei entgegen."

4. Der Surrealismus. Im Zwischenreich der Dichtung, Malerei und Psychologie. Was wollten die Surrealisten? Eine Frage an Breton, Aragon und Soupault. Das Phänomen des italienischen Futurismus: Der 1. Durchbruch surrealistischer Motive. Die Manifeste Marinettis. „Modernolatria“ und das „periculose vivere ${ }^{\star}$. Nachklänge Nietzsches und Vorklänge des Surrealismus. - Der 2. Durchbruch auf dem Weg zum Surrealismus: die Zürcher Dada-Bewegung von 1916. Was war "Dada"? Ein Künstlerjux mit politischen und metaphysischen Hintergründen. Drei Rüdkblicke der Gründer: Tristan Tzara, Hans Arp, Richard Huelsenbeck. - Die 3. Etappe: das Ende Dadas und die Geburt des Surrealismus in Paris. - Die surrealistische Zentrale und die Tätigkeit der neuen Gruppe. Das ewige Dilemma: die Unlösbarkeit des Problems Kunst und Politik. Die Faszination durch die russische Revolution. Die entscheidende Frage von Pierre Naville: Engagement der Surrealisten in der KPF oder Unabhängigkeit von der Parteidisziplin? Am Ende: der Ausschluß aus der KPF. - Vom Recht, "nein" zu sagen. - Von der surrealistischen Dichtung und der „écriture automati$q u e^{\alpha}$. Der verborgene Platonismus. Im Bann des Orients. Die Adressen an den Dalai Lama, an die Rektoren der europäischen Universitäten und an den Papst von Rom. - Surrealistische Malerei. Ein Blidk zu den Quellen: zurück über den Zöllner Rousseau, Victor Hugos Tuschen, die Romantik mit Füßli und Blake - und weiter zurück über Goya zu Leonardo da Vinci und seinen Geheimnissen nim alten Mauerwerk“; der Gang unter der 
Führung Freuds in die Tiefenschichten der Seele und die Praehistorie der Menschheit. - Die Philosophie der "coincidentia oppositorum". - Wesenszüge surrealistischer Malerei: Kunst im zerbrochenen Spiegel der Welt. Die "ver-rüdkten" Gegenstände. Eine Katachrese der Malerei. Das Wunderbare und vom "Geheimnis, daß der Mensch Kunst macht". Vom Surrealismus zur COBRA-Gruppe. - Ausblids.

Kapitel IV Hinter dem Vorhang der Zeitgeschichte. Strömungen, Theorien, Antagonismen der Zeit: Ihr Widerspiel auf dem Theater des XX. Jahrhun-

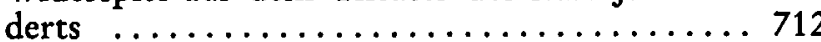

1. Vorbemerkungen. Theater im 20. Jahrhundert. Antagonismen und Mehrschichtigkeit. Die Offnung zu neuen Horizonten. Vor dem Ersten Weltkrieg: die „Moderne" beginnt. "L'Entre-deux-guerres"; eine frudhtbare und erregend-freche Generation. Drei Ereignisse des Theaterlebens: 1. Die Schöpfung der 7. Kunst. Von Méliès zu Cocteau. 2. Das Ballet - auf dem Wege zum totalen Theater. Zusammenarbeit von Dichtern, Malern, Musikern. 3. Die Herrschaft der Regisseure. Das „Kartell der Vier“ und die Wirksamkeit der großen ausländischen Theatermänner: Stanislawski, Appia, Craig, Reinhardt. Theater als Interpretation eines literarischen Werkes oder Theater als autonome Kunst?

2. Vier große Exponenten der französischen Dramaturgie. Gemeinsamkeiten und Gegnerschaft:

Antonin Artaud. "Le théâtre et son double - "die unbestreitbar bedeutendste Theaterschrift des 20. Jahrhunderts". - Die Sprache der Gestik Wortsprache und Zeichensprache. Der Eindruck des Bali-Theaters. "Théâtre pur “ und "Théâtre de la Cruauté ${ }^{\star}$. Was heißt Grausamkeit? Anklänge an Schopenhauers Metaphysik des Leidens. - Der ideale Spielsaal und die "musikalische Partitur" als Notation einer universellen Theatersprache. Artauds Theateraufführungen und Strindbergs Traumspiel.

Paul Claudel. Die Faszination durch das Musiktheater. Von der Zusammenarbeit Claudel-Milhaud. Kritik an der klassischen Oper Gludk - Mozart und ihre Begründung. Claudels Auseinandersetzung mit dem Musikdrama Wagners. Die Wendung zum japanischen Theater. Claudel und die NôSpiele. Versuch einer Adaptation in einem biblischen Stück. Grundlegende dramatische Gedanken unter dem Einfluß des fernöstlichen Theaters: vom Tanz und vom Chor, vom Orchester und vom Bühnenbild, von der Gestik und von der Bedeutung des Kostüms. - Romantischer Nachschein letzter Orientträume? Der nachclaudelsche Osten.

Jean Paul Sartre. Ein Philosoph vom Fach als Theaterdichter und Drehbuchautor. Unterschied zu Artaud, Claudel, Ionesco. Der marxistische Nährboden seiner Stüdke. Existenzialismus und Marxismus. „L'existencialisme est un humanisme". Umstülpung der platonisch-christlichen Ordnung der essentia und existentia; der radikale Anti-Idealismus und Atheismus; espoir und dés-espoir. "Wir wollen nichts von unserer Zeit verpassen." 
Wie Sartre zum Marxismus kam. Ein autobiographischer Text. Sartres Kritik am Marxismus und die Hoffnung auf einen neuen Sinn des Existenzialismus als einer Form "verstehender Erkenntnis" in einer sozialen Welt. - Analyse von 3 Stücken: Die ${ }_{„} M o u c h e{ }^{\alpha}{ }^{\alpha}$ oder von der Gideschen Disponi. bilität zum sozialen Engagement; die ${ }_{n} H u i s \operatorname{clos}^{*}$ oder ${ }_{n}$ der Prozeß der fortschreitenden Aufhebung der Selbsttäuschung"; "Le Diable et le bon Diew oder eine Sartresche Fausttragödie. - Sartre im Feuer einer vierfachen Kritik: die Antimarxisten einer bürgerlichen Tradition, die antiatheistischen Existenzialisten christlicher Prägung, die Strukturalisten als Anti-Humanisten, die Anti-Ideologen als Liberalisten.

Eugène lonesco. Erinnerung an Schiller: „Es ist höchste Zeit, daß ich die philosophische Bude schließe." Ionesco: Fort von den Ideologien, zurück zum reinen Theater. Eine Anekdote aus England oder die Klärung der Ionescoschen Position. "Tout n'est pas de la politique". Die Geschichte als kontinuierliche Folge von Verirrungen und die Ideologie als Alibi der Unwahrhaftigkeit. Von der Insuffizienz der Literatur in unserer Zeit. Hat es noch einen Sinn zu schreiben? Das „étonnement primordial". - Die Funktion des Theaters. Faszinosum des Marionettentheaters. Die aktuelle Inaktualität. Ionesco: Glied einer Tradition von Hiob über Sophokles zu Shakespeare und dem Anti-Theater. Von der Struktur seiner Theaterstücke im Bilde eines Bildes. Die coindidentia oppositorum als philosophische Anschauung von Tragödie und Komödie. Der Gegensatz zu Sartre.

3. Rückschau und Blick in die Gegenwart. - Charakteristika der heutigen kulturellen Erscheinungen. - Die "Anti“'Strömungen. -

Bibliographische Orientierung $\ldots \ldots \ldots \ldots \ldots \ldots \ldots \ldots 771$

Namenverzeichnis ..................... 806 